\title{
Application of the Concept of Nuclear ud Pair Clusters
}

\author{
Abdul L. Bhuiyan ${ }^{1}$ \\ ${ }^{1}$ Box 13, Head Post office, Comilla-3500, Bangladesh \\ Correspondence: Abdul L. Bhuiyan, Box 13, Head Post office, Comilla-3500, Bangladesh. E-mail: \\ ananda.computers@yahoo.com
}

Received: December 15, 2015 Accepted: February 4, 2016 Online Published: February 20, 2016

doi:10.5539/apr.v8n2p29

URL: http://dx.doi.org/10.5539/apr.v8n2p29

\begin{abstract}
In a previous paper, the author of this paper presented a model in which it was proposed that the nuclei consist of clusters of ud pairs, where $u$ and $d$ are $u$ quark and d quark, respectively. In this paper, the author has used the model in elucidating several phenomena, such as binding of $u$ and $d$ in $u d$ by the exchange of electron-positron pairs or photons between them, energy of the exchanged photons, nature of forces between $u$ and $d$, interaction of energetic photons with nuclear ud pairs, existence of nuclear quadrupole moment, creation of dark matter via simultaneous increase of $\mathrm{h}$ and decrease of $\mathrm{c}$ as the universe expands, etc.
\end{abstract}

Keywords: ud pairs, dark matter, quantum gravitational force, laser beams

\section{Introduction.}

With a view to establish relationship between an infinite number of partons and the three quarks inside the nucleon, Sekine (Sekine, 1985, 2012) applied the cantor set (Cantor, 1883) to an infinite number of point-like quarks and antiquarks constituting the nucleon and the dark matter and has shown, through the introduction of color charges in QCD, that the theory of the strong interaction (color force) does not exist between quarks and antiquarks. Since one quark has only one antiquark, and if, according to Sekine (Sekine, 2012), the embryo universe contains quarks and antiquarks only, then the principle of probability demands that the number of quarks should be equal to the number of antiquarks. For this, the quarks should be equal to the number of antiquarks. For this, the quarks and antiquarks would have annihilated each other, leaving the embryo universe completely filled with radiations; that is, there would be no matter left in it. It is against this back-ground that any model describing the composition of the embryo universe in terms of quarks and antiquarks just before the expansion should be untenable.

Protons and neutrons are made up of $u$ quarks and d quarks only. Particles made up of other quarks decay very rapidly into protons and neutrons, indicating that the $u$ quarks and the $d$ quarks are most stable of all quarks. The factors contributing to the stability of $u$ quarks and d quarks in protons and neutrons may be worked out in the following way.

The emission of beta particles (electrons) by naturally occurring radioactive nuclei is attributed to the spontaneous transformation of a neutron into a proton, an electron, and an antineutrino; thus

$$
\text { udd } \rightarrow \text { uud }+\mathrm{e}^{-1}+v^{\prime} \quad \text {....... }
$$

A neutron, dud, may be represented by either of two canonical structures (du) $d$ and $d$ (ud). In either structure, one $\mathrm{d}$ quark is paired up with the $\mathrm{u}$ quark while the other remains unpaired, for which one may presume that it is this unpaired d quark which emits an electron and an antineutrino. It is also found that radioactive elements obtained in the laboratory emit a positron and a neutrino during the transformation of a proton into a neutron; thus

$$
\text { uud } \rightarrow \text { udd }+\mathrm{e}^{+1}+v
$$

A proton, udu, may be represented by either of two canonical structures- (ud)u and $u(d u)$. In either structure, a $u$ quark remains transiently unpaired with the d quark. Therefore, this unpaired u quark emits a positron and a neutrino before being transformed into a d quark, indicating that an unpaired $\mathrm{u}$ quark is not stable; it tends to stabilize by forming a pair with a d quark. All this indicates that isolated $\mathrm{u}$ quarks and $\mathrm{d}$ quarks tend to stabilize themselves by forming pairs of composition ud. This prompted us to present a model in which the nuclear structures are described in terms of clusters of ud pairs (Bhuiyan, 2013). Some features of the paper are used 
here to help elucidate several phenomena as the mechanism of formation of ud pairs, nature of the forces between $u$ and $d$ in $u d$, exchange of electron- positron pairs or photons between $u$ and $d$ in $u d$, energy of the exchanged photons, interaction of energetic photons with nuclear ud pairs, existence of nuclear quadrupole moment, creation of dark matter, etc.

\section{Formation of Quarks}

Sekine (Sekine, 2012) maintains that pairs of quarks were formed in the first moments after the Big Bang. But some properties of quarks indicate the presence of other particles in the early universe before the appearance of quarks. For example, equation (3) shows that an unpaired d quark emits an electron during transformation into a u quark.

$$
\mathrm{d} \rightarrow \mathrm{u}+\mathrm{e}^{-1}
$$

Similarly, an unpaired $\mathrm{u}$ quark transforms into a $\mathrm{d}$ quark with the emission of a positron; thus

$$
\mathrm{u} \rightarrow \mathrm{d}+\mathrm{e}^{+1}
$$

Equations (3) and (4) indicate that the interconversion of $u$ quark and d quark is a cyclic process. So, substituting the value of $u$ quark from equation (4) into equation (3), one obtains

$$
\mathrm{d} \rightarrow \mathrm{d}+\left(\mathrm{e}^{+1}+\mathrm{e}^{-1}\right)
$$

Similarly, substituting the value of $d$ quark from equation (3) into equation (4), one obtains

$$
\mathrm{u} \rightarrow \mathrm{u}+\left(\mathrm{e}^{-1}+\mathrm{e}^{+1}\right)
$$

If the processes (3) through (6) are continued, it will be found that $u$ quarks and d quarks are made up of "layers" of positrons and electrons much like a spherical onion (Figure 1). In the case of d quark, the outermost layer is formed by an electron. This will be followed by the layer formed by the positron. This order of arrangement will be followed throughout the quark. In the case of $u$ quark, the outermost layer is formed by a positron which will be followed by the layer consisting of an electron. This order of arrangement will be obeyed throughout the quark. Under these arrangements, a d quark will always emit an electron and a u quark will always emit a positron. Since u quarks and d quarks are made up of electrons and positrons, it is the latter particles, that is, electrons and positrons, which had appeared before the appearance of quarks.

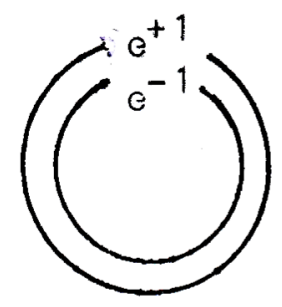

(a) u Quark

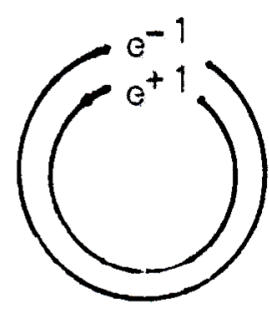

(b) d Quark

Figure 1. Constitution of (a) u Quark and (b) d Quark

It is believed that the early (or embryo) universe was infinitely hot. At such high temperature, the particles (electrons and positrons) were moving randomly with extremely high speed. Due to mutual (elastic) collisions, a highly chaotic and disorderly state was created. Let us call this state the state -1 and the state in which the particles occupied orderly positions in quarks the state-2. In state-1, the particles also possess disorderly random spins. As the universe starts cooling, the particles start losing kinetic energy and speed. As a result, the particles start dropping from state-1 into state-2 through spin transition. This may be elucidated by the application of Boltzmann's law in the form

$$
\mathrm{n}_{\mathrm{u}} / \mathrm{n}_{1}=\mathrm{e}^{-\triangle \mathrm{E} / \mathrm{kT}}
$$

Where $n_{u}=$ population of the particles in the upper level (state-1),

$\mathrm{n}_{1}=$ population of the particles in the lower level (state-2)

$\triangle \mathrm{E}=$ energy separation between the two states,

$$
\mathrm{k}=\text { Boltzmann constant. }
$$

Taking the natural logarithm of equation (7), we have 


$$
\ln \left(\mathrm{n}_{\mathrm{u}} / \mathrm{n}_{\mathrm{l}}\right)=-\triangle \mathrm{E} / \mathrm{kT}
$$

or,

$$
\ln \left(\mathrm{n}_{\mathrm{l}} / \mathrm{n}_{\mathrm{u}}\right)=\triangle \mathrm{E} / \mathrm{kT}
$$

As the universe cooled, $\mathrm{T}$ decreased, for which the magnitude of $\triangle \mathrm{E} / \mathrm{kT}$ increased. As a result, the population of electrons and positrons in state-2 increased and the particles arranged themselves in the orderly fashion which we have described above. In this way, the quarks came into being from electrons and positrons.

Simultaneous with the formation of quarks, another event might occur. During cooling, some electrons and positrons might come in close proximity to each other and formed pairs through spin correlation. Since an electron and a positron are antiparticle to each other, the pairing may lead to mutual annihilation into photons. However, these photons might exist in reversible equilibrium with electron- positron pairs. All this indicates that electrons, positrons and photons had appeared before the appearance of quarks. As a matter of fact, electrons and positrons are the precursors of quarks.

\section{Pairing of u Quarks with d Quarks.}

Let us return to figure-1. When an isolated u quark comes in close proximity to an isolated $d$ quark, then an electron emitted from the outermost layer of the $d$ quark is captured by the $u$ quark while simultaneously a positron emitted from the outermost layer of the u quark is captured by the d quark. Thus, an electron- positron pair is exchanged between the two quarks. As a result, the u quark and the d quark undergo transformation into $d$ quark and u quark, respectively. But the resulting structure becomes different from the initial one. In order to make the final structure identical with the initial one, the newly formed $u$ quark may emit a positron which is captured by the newly formed d quark, while simultaneously the latter may emit an electron which is captured by the former. In this way, the initial structure becomes identical with the final one when a total of two electronpositron pairs are exchanged between the $u$ quark and the $d$ quark. If one electron- positron pair forms a single bond, then two electrons - positron pairs will form a double bond. Then the two quarks may form a pair represented by

$$
\begin{gathered}
u+d \rightarrow u=d \\
x(u+d) \rightarrow x(u=d)
\end{gathered}
$$

in which $\mathrm{x}$ number of $\mathrm{u}$ quarks unite with $\mathrm{x}$ number of $\mathrm{d}$ quarks to produce $\mathrm{x}$ number ud paris and $\mathrm{x}=1,2$, 3,

Besides forming covalent (double) bond, the two quarks are likely to form ionic bond as is shown in equation (12).

$$
\mathrm{x}\left(\mathrm{u}^{+2 / 3}+\mathrm{d}^{-1 / 3}\right) \underset{\mathrm{x}\left(\mathrm{u}^{+2 / 3} \cdot \mathrm{d}^{-1 / 3}\right) \quad \cdots \cdots}{\rightarrow \mathrm{x}(\mathrm{ud})}
$$

Equation (13) represents the formation of ionic-covalent bond between the u quark and d quark. In presence of an initiator, such as an isolated u quark or an isolated d quark, the product ud in equation (10) may undergo polymerization as in shown in equations (14) and (15).

\section{Photons in Nuclei}

$$
\begin{aligned}
\mathrm{x}(\mathrm{ud}) & \rightarrow-\mathrm{u}-\mathrm{d}-\mathrm{u}-\mathrm{d}-\mathrm{u}-\mathrm{d}- \\
& \rightarrow(-\mathrm{u}-\mathrm{d}-)_{\mathrm{x}} \quad \ldots \ldots . . .
\end{aligned}
$$

In the polymer represented by equation (15), a u quark is bonded to a d quark through a single bond which is constituted by an electron - positron pair. Since an electron and a positron move through the same region of space between $\mathrm{u}$ and $\mathrm{d}$ in exactly opposite directions, they are likely to undergo mutual annihilation into photons. The electron and positron possess the same rest mass, $\mathrm{m}_{0}$. So, the annihilation of an electron- positron pair may result in a loss of $2 \mathrm{~m}_{0}$ gram which is equivalent to the energy given by

$$
\begin{aligned}
\mathrm{E} & =2 \mathrm{~m}_{\mathrm{o}} \mathrm{c}^{2} \text { ergs } \quad \ldots \ldots \ldots \ldots . . . \\
& =2 \times 9.11 \times 10^{-28} \times\left(2.998 \times 10^{10}\right)^{2} \mathrm{ergs} \quad \ldots . . \\
& =1.02 \mathrm{MeV} \ldots \ldots \ldots .
\end{aligned}
$$

Conservation of momentum requires that the energy equal to $1.02 \mathrm{Mev}$ should represent two photons with zero net momentum; that is, the two photons must move in exactly opposite directions. This is in agreement with the literature report that the energy equal to $1.02 \mathrm{MeV}$ appears commonly as two photons moving in exactly opposite directions (Glasstone, 1958). Each photon would possess energy equal to $0.51 \mathrm{MeV}$ which is equivalent 
to $\mathrm{m}_{0} \mathrm{c}^{2}$ of one electron or one positron. So, like the electron - positron pair, two corresponding photons moving in exactly opposite directions may be considered to be exchanged between the $u$ quark and d quark, suggesting that an electron- positron pair exists in reversible equilibrium with two photons. For this, we may consider the exchange process either in terms of electron- positron pair or in terms of two photons for a single bond.

Let us return to equations (1) and (2) in which neutrino and antineutrino are emitted simultaneously with positron and electron, respectively. If the exchanged electron - positron pair exists in reversible equilibrium with the photons, then both the electron-positron pair and the photons should be equally stable. One way in which the stability between an electron and a positron may be established is to bind them with a pair of neutrinos as follows. The electron may emit an antineutrino which is captured by the positron and simultaneously the positron may emit a neutrino that is captured by the electron. In this way, the electron and positron may be bonded to each other through the exchange of pair of neutrinos between them. As the photons are the products of annihilation of an electron-positron pair, the bond constituted by the neutrinos between the electron and positron should continue to exist between the pair of product photons. Thus, the exchanged photons stabilize themselves through the neutrino bond. The association of the neutrinos with photon, electron and positron shows that these particles may move with speed which is comparable to the speed of light.

\section{Energy of an Exchanged Photon.}

Let us confine a ud pair in a one-dimensional box of length $\mathrm{AB}(=\mathrm{a})$ in a nucleus (Figure 2.). The u quark and $\mathrm{d}$ quark are situated at the points $\mathrm{A}$ and $\mathrm{B}$, respectively. The positron emitted by the $\mathrm{u}$ quark moves along $\mathrm{AB}$ toward the point $\mathrm{B}$ and is captured by the $\mathrm{d}$ quark there. Simultaneously, the electron emitted by the $\mathrm{d}$ quark moves along BA toward the point $\mathrm{A}$ and is captured by the $\mathrm{u}$ quark there. As an electron and a positron possess the same rest mass, to an outside observer, they will appear identical and indistinguishable, for which the observer will find that a particle (photon or the electron/ positron) performs a round trip travel between the two quarks through the distance 2a. The wavelength associated with the wave of an exchanged photon must fit the distance $2 \mathrm{a}$. If $\lambda$ be the wavelength associated with a photon, then, for constructive interference, the distance $2 \mathrm{a}$ should be given by $1 \lambda, 2 \lambda, 3 \lambda, \ldots, \mathrm{n} \lambda$, where $\mathrm{n}$ is a positive integer. In general, we have

$$
\begin{aligned}
2 \mathrm{a} & =\mathrm{n} \lambda \\
\text { Or, } \mathrm{a} & =\mathrm{n} \lambda / 2 \quad \ldots \ldots .
\end{aligned}
$$

As the photon possesses wave-particle duality, one may substitute $\lambda$ in equation (20) with $h / p$, where $p$ is the momentum of the particle, to obtain

$$
\mathrm{p}=\mathrm{nh} / 2 \mathrm{a}
$$

Raising both sides of equation (21) to second power and then dividing by $2 \mathrm{~m}$, where $\mathrm{m}\left(=\mathrm{E} / \mathrm{c}^{2}\right)$ is the mass of the photon, one obtains

$$
\begin{aligned}
& \mathrm{p}^{2} / 2 \mathrm{~m}=\frac{\mathrm{n}^{2} \mathrm{~h}^{2}}{8 m \mathrm{a}^{2}} \\
& \text { Or, } \mathrm{E}=\frac{\mathrm{n}^{2} \mathrm{~h}^{2}}{8 m \mathrm{a}^{2}} \quad \ldots \ldots . . .
\end{aligned}
$$

where $\mathrm{E}$ is the kinetic energy of the exchanged photon. Equation (23) gives the energy of a photon due to the presence of one ud pair. If there are $x$ number of ud pairs in the nucleus, then the total energy of $x$ number of exchanged photons in the nucleus is given by $\mathrm{xE}$. But there are $\mathrm{x}$ pairs of photons in $\mathrm{x}$ number of ud pairs. So, the total energy of $x$ pairs of photons in $x$ number of ud pairs is given by $x(2 E)$.

\section{A} B

Figre 2. One-dimensional box $\mathrm{AB}$ in which the ud pair is confined.

\section{The Nature of the Forces Between $u$ and $d$.}

Let us return to Figure 2. The u quark and d quark are situated at the points $\mathrm{A}$ and $\mathrm{B}$, respectively, of the one-dimensional box $\mathrm{AB}(=\mathrm{a})$. An exchanged photon moves in this box with quantum mechanical momentum, $\mathrm{p}$, given by equation (21). But we evaluated this momentum taking into consideration the motion of the particle in space coordinates only. Let us now include the time coordinate with the motion of the particle. Since AB is of 
magnitude a, a photon emitted by u quark and captured by d quark moves through the distance a with speed equal to $a / t$, where $t$ is the time in which the photon arrives at $d$ from $u$ without making a round trip travel. Since $\mathrm{m}$ is the mass of the photon, its momentum is given by

$$
\mathrm{p}_{\mathrm{c}}=\mathrm{ma} / \mathrm{t} \quad \text {....... }
$$

where c stands for classical. As the classical momentum should be equal to the quantum mechanical momentum, we have

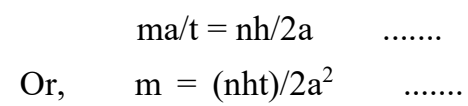

Multiplying equation (26) with $\mathrm{c}^{2}$, one obtains the energy of an exchanged photon given by

$$
\begin{aligned}
\mathrm{E} & =\mathrm{mc}^{2} \quad \ldots \ldots . . \\
& =\left(\mathrm{nhtc}^{2}\right) / 2 \mathrm{a}^{2} \quad \ldots . .
\end{aligned}
$$

The force carried by a photon may be obtained by dividing equation (28) by a; thus

$$
\begin{gathered}
\mathrm{F}=\mathrm{E} / \mathrm{a} \quad \ldots \ldots . \\
=\left(\mathrm{nhtc}^{2}\right) / 2 \mathrm{a}^{3} \quad \ldots \ldots . .
\end{gathered}
$$

The total force carried by both the photons together is given by

$$
\mathrm{F}_{\mathrm{t}}=\left(\mathrm{nhtc}^{2}\right) / \mathrm{a}^{3}
$$

Equations (26), (28), and (30) show that the mass, energy, and force carried by a photon between $u$ and $d$ are spacetime quantized.

Let us return to equation (26) and rearrange it to obtain

$$
\mathrm{a}^{2}=(\mathrm{nh} / 2 \mathrm{~m}) \mathrm{t}
$$

Equation (32) is quadratic in a, so we describe the motion of an exchanged photon in a two-dimensional space-time (Figure 3). The straight lines $\mathrm{OB}$ and $\mathrm{OY}$ lie on the $\mathrm{X}$ axis and $\mathrm{Y}$ axis, respectively, while $\mathrm{O}$ forms the origin of coordinates. The straight line AOB represents a one-dimensional box of length equal to a. Let the $u$ quark and d quark be situated at the points A and B, respectively, so that the two quarks are separated by the distance a. Using equation (32), one may plot a along the $\mathrm{Y}$ axis and $\mathrm{t}$ along the $\mathrm{X}$ axis to obtain the parabolic curve COF or EOD. Let a photon, belonging to d quark; be situated at the point $\mathrm{C}$ at an instant. The photon is acted upon by two attractive forces, $F_{1}$ and $F_{2}$, exerted by the center of $u$ quark and the center of $d$ quark, respectively. Under the action of $F_{1}$, the photon tends to move in a direction parallel to the $\mathrm{X}$ axis, while under $\mathrm{F}_{2}$, it tends to move in a direction parallel to the $\mathrm{Y}$ axis. As a result, the photon will move toward the point $\mathrm{O}$ along the curved path $\mathrm{CO}$, thus describing a parabolic path. Actually, in the one-dimensional box AOB, the path of motion of the photon from $\mathrm{d}$ quark to $\mathrm{u}$ quark should be along the straight line $\mathrm{BO}$ which is the shortest possible distance from $\mathrm{B}$ to $\mathrm{O}$. Thus, the photon projected horizontally from the point $\mathrm{C}$ falls to the point $\mathrm{O}$ in a parabolic path in spacetime. Similarly, the other photon belonging to the u quark and situated at the point D may travel toward the point $\mathrm{O}$ through the shortest possible distance $\mathrm{AO}$ in a curved path DO in spacetime. According to Einstein's general theory of relativity, gravity is manifested by the motion of objects along the paths that follow the shortest possible distance in a curved spacetime and such motion has also been shown to be a parabola (Feynman, Leighton, \& Sands, 1988). Thus, the forces, $F_{1}$ and $F_{1}$, acting on the exchanged photons are of the nature of gravity. This implies that

where, $\mathrm{m}=\left(\mathrm{nht} / 2 \mathrm{a}^{2}\right)$ and $\mathrm{g}=\mathrm{c}^{2} / \mathrm{a}$.

$$
\begin{aligned}
\mathrm{F}_{1} & =\mathrm{F}_{2}=\left(\mathrm{nhtc}^{2}\right) / 2 \mathrm{a}^{3} \\
= & (\mathrm{nht}) / 2 \mathrm{a}^{2}\left(\mathrm{c}^{2} / \mathrm{a}\right) \\
= & \ldots \ldots \ldots
\end{aligned}
$$

It is found that gravity attracts gravity (Goldman, Hughes, \& Nieto, 1988). This indicates that $F_{1}$ and $F_{2}$ interact to produce gravitational force of attraction; thus

$$
\begin{aligned}
\mathrm{F}_{\mathrm{G}}=\mathrm{F}_{1}+\mathrm{F}_{2} & =2 \mathrm{mg} \quad \ldots \ldots . \\
& =\left(2 \mathrm{c}^{2} \mathrm{a} / \mathrm{m}\right)\left(\mathrm{m} \cdot \mathrm{m} / \mathrm{a}^{2}\right)
\end{aligned}
$$




$$
=\mathrm{G}\left(\mathrm{m} \cdot \mathrm{m} / \mathrm{a}^{2}\right)
$$

where $\mathrm{G}$ is the gravitational force constant and $\mathrm{F}_{\mathrm{G}}$ represents the gravitational force of attraction between the two exchanged photons. The force $\mathrm{F}_{\mathrm{G}}$ carried by the two exchanged photons keeps the $\mathrm{u}$ quark and $\mathrm{d}$ quark in ud bound together. However, it is evident that gravity and gravitational force of attraction are spacetime quantized.

There exists another kind of force of attraction between the positively charged $u$ quark and the negatively charged $d$ quark. This is called Coulombic force, $F_{c}$, whose strength is proportional to the product $\mathrm{q}_{1} \mathrm{q}_{2}$ and inversely proportional to the square of the distance a between the $u$ quark and d quark, where $q_{1}$ is the charge carried by one quark and $\mathrm{q}_{2}$ is the charge carried by the other quark. Stating mathematically

$$
\mathrm{F}_{\mathrm{c}}=\left(\mathrm{q}_{1} \mathrm{q}_{2}\right) / \mathrm{a}^{2}
$$

The gravitational mass $\mathrm{m}$ is considered as a charge; an object is said to feel a gravitational force in proportion to its gravitational mass, just as it would feel an electromagnetic force in proportion to its electric charge (Goldman, Hughes, \& Nieto, 1988). This implies that the quantity (m.m) in equation (38) should be the same as that of the product $\mathrm{q}_{1} \mathrm{q}_{2}$ in equation (39). This is further supported by the concept that at the grand unification energy, where quarks, electrons, and positrons would all be the same, all forces would have the same strength (Hawking, 1988). So, the two forces, $\mathrm{F}_{\mathrm{G}}$ and $\mathrm{F}_{\mathrm{c}}$, can have the same strength on condition that

$$
\mathrm{G}=1
$$

Substituting $\mathrm{G}$ with its value from equation (37), one obtains (41)

$$
\left(2 c^{2} a\right) / m=1
$$

Substituting $\mathrm{m}$ with its value from equation (26) and then simplifying, one obtains

$$
\mathrm{h} / \mathrm{c}^{2}=(4)(1 / \mathrm{n})\left(\mathrm{a}^{3} / \mathrm{t}\right)
$$

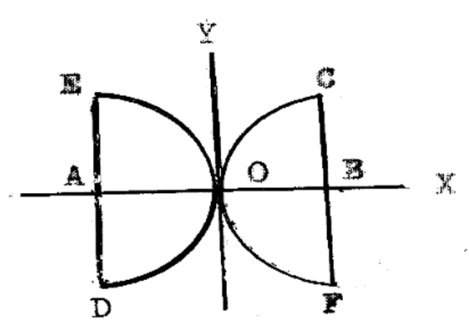

Figure 3. Paths (COF and EOD) in space time followed by a particle exchanged between u quark and d quark of the ud pair

\section{The Dark Matter}

It is believed that the universe was intensely hot at the beginning. At this high temperature, the quarks were moving around with a very high speed, for which they suffered elastic collisions with each other. As soon as cooling started, the speed of the quarks started to decrease. As the cooling proceeded, the quarks, especially the $u$ quarks and d quarks, started to suffer inelastic collisions during which they formed the ud pairs. Since these pairs were not fairly stable, polymerization, or clustering, of them began leading to the formation of larger nuclei; that is, smaller nuclei started to cluster together to form the larger ones. As a result, the total volume of the nuclei started to increase. Let the quantity $\mathrm{a}^{3} / \mathrm{t}$ containing electrons, positrons, photons and quarks be proportional to the size of the embryo universe.

Since $\mathrm{h}$ and $\mathrm{c}$ in equation (42) are constant quantities, the ratio $\mathrm{h} / \mathrm{c}^{2}$ is a constant quantity. This implies that the right side of the same equation should be a constant quantity, suggesting that the universe was in the static condition at the beginning. So when God wanted to create the universe. He said "BE" and instantly the universe came into being. Since then the universe remained static. But there are evidences which support the expansion of the present universe. Let us return to equation (26) and rearrange it to obtain

$$
\mathrm{H}=\left(2 \mathrm{ma}^{2} / \mathrm{t}^{2}\right) \mathrm{t} / \mathrm{n}
$$

where $2 \mathrm{~m}$ = gravitational mass of the particles which existed in the hot early universe before expansion.

$\mathrm{a}=$ distance between the interacting particles or the length of the one-dimensional universe, 
$\mathrm{t}=$ time in which a particle travels through the distance $\mathrm{a}$,

$\mathrm{n}=\mathrm{a}$ positive integer.

Then the gravitational energy of the particles is given by

$$
E_{G}=\left(2 m a^{2}\right) / t^{2}
$$

From equations (43) and (44), one obtains

$$
\mathrm{h}=\left(\mathrm{E}_{\mathrm{G}} / \mathrm{n}\right) \mathrm{t}
$$

Thus, $\mathrm{h}$ is directly proportional to the gravitational energy of the particles of the embryo (early) universe. Equation (45) further states that $\mathrm{h}$ changes with time.

It has been stated in section-2 that electrons and positrons were the precursors of quarks and that the electronpositron pairs existed in reversible equilibrium with photons in the embryo universe of four- dimensional space time volume $\mathrm{a}^{3} / \mathrm{t}$ given in equation (42). So, we may assume that electrons, positrons and photons were the only particles present in the embryo universe prior to the appearance of quarks. As electron-positron pairs existed in reversible equilibrium with photons, the total mass of the photons should be equal to the total mass of the electron- positron pairs. Therefore, if $\mathrm{M}$ be the total mass of the photons, then the total energy of the photons should be given by

$$
\begin{aligned}
& \mathrm{E}=\mathrm{Mc}^{2} \quad \ldots \ldots . . \\
& \text { or, } \quad \mathrm{c}^{2}=\mathrm{E} / \mathrm{M}
\end{aligned}
$$

Where $\mathrm{c}$ is the speed of light. Since the rest mass of light photon is negligibly small, the magnitude of $\mathrm{M}$ in equation (47) should be negligibly small compared to the magnitude of $\mathrm{E}$. This implies that $\mathrm{c}^{2}$ is proportional to E. So, substituting $\mathrm{c}^{2}$ with $\mathrm{E}$ in equation (42) and inserting the value of $\mathrm{h}$ from equation (45) into the same equation, one obtains.

$$
\begin{gathered}
\left(E_{G} / n\right) t / E=(4)(1 / n)\left(a^{3} / t\right) \\
\text { or, }\left(E_{G} / n\right) /(E / t)=(4)(1 / n)\left(a^{3} / t\right)
\end{gathered}
$$

where $E_{G} / n=$ quantized gravitational energy and $E / t=$ time rate of change of the energy of the photons.

Let $\mathrm{t}$ be negligibly small (not exactly zero) at the beginning of cooling when $\mathrm{E}$ is extremely large. As cooling starts, $t$ starts to increase, for which $E / t$ decreases; that is, $E$ decreases due to decrease of kinetic energy and speed of the photons. According to the principle of conservation of energy, the decrease of $\mathrm{E}$ transforms into increase of $E_{G}$. As a result, $n$ decreases. The decrease of $n$ causes the size of the universe, $a^{3} / t$, to increase. Thus, the universe starts to expand as soon as cooling starts. In a nutshell, as the cooling starts, the kinetic energy and speed of the photons start to decrease while the universe drops from higher energy levels into the lower energy ones and simultaneously the gravitational energy starts to increase. As a result, the universe starts to expand. This process continues till the universe arrives at the ground level $(n=1)$. This period of the universe may be called the period of quantum mechanical state, or the period of inflationary expansion. As soon as $n=1$, the quantum mechanical state of the universe ends and its cosmological state begins. But the increase of $E_{G}$ continues till the energy and speed of the photons become negligible. All this indicates that the gravitational energy, $E_{\mathrm{G}}$, may be considered as a candidate for the dark matter.

Let us return to equations (37) and (38) and write

$$
\mathrm{G}=2 \mathrm{c}^{2} \mathrm{a} / \mathrm{m}
$$

Substituting $\mathrm{m}$ with its value from equation (26) and then simplifying, one obtains

$$
\mathrm{G}=\left(4 \mathrm{c}^{2} / \mathrm{h}\right)(1 / \mathrm{n})\left(\mathrm{a}^{3} / \mathrm{t}\right)
$$

The quantity $G$ was equal to 1 immediately after the Big Bang when $F_{G}$ and $F_{c}$ were equal and the expansion of the universe had not as yet started. As soon as the universe started to expand, $\mathrm{a}^{3} / \mathrm{t}$ started to increase causing $\mathrm{G}$ to increase. As a result, $F_{G}$ started to be greater than $F_{c}$. It is at this stage when the two forces started to separate. Also, the distance a of interaction between $u$ and $d$ increases with the increase of $a^{3} / t$, for which the magnitude of the nuclear force decreases. In consequence, the nuclei become more and more unstable.

\section{Interaction of Energetic Photons with Nuclear ud.}

A nucleus is bombarded with an outside projectile consisting of an energetic photon. Since the nucleus is supposed to consist of ud pairs, the projectile may collide with one of these pairs. During collision, the energetic photon may materialize into an electron-positron pair (Beiser, 1987); thus 


\section{Photon $\underset{\text { nucleus }}{\longrightarrow} \mathrm{e}^{-}+\mathrm{e}^{+} \quad \ldots \ldots$}

It has been shown earlier above that an electron-positron pair may constitute one of the two bonds between $u$ and $\mathrm{d}$ in the ud pair. So, the collision of the energetic photon with a ud pair implies the collision of one electron-positron pair with another of the same kind. The collision may set free the electron-positron pair of one of two bonds between $u$ and $d$, provided that the energy of the energetic photon exactly matches the bond energy. Immediately after the scission of the bond, the electron of the energetic photon may pick up the positron of the scissioned bond, while simultaneously the positron of the energetic photon may pick up the electron of the scissioned bond. As a result, two electron-positron pairs equivalent to four photons are liberated. So, the emitted radiation then consists of two pairs of photons of the same frequency and the same wavelength. Since the incident energetic photon consists of one pair of photons (or one electron-positron pair), and the emitted radiation bundle consists of four photons, it may be concluded that the energetic photon has cloned itself. However, all the four photons in the emitted radiation will move in the same direction after collision. The emitted radiation may exhibit the following characteristic properties.

a) It may exhibit wave-particle duality.

b) The constituent photons, or the electron-positron pairs, may possess spins.

c) It may possess two electron-positron pairs - two negatively charged electrons and two positively charged positrons - that may produce two dipoles or one quadrupole.

d) The electrons may make them behave as matter particles and the positrons may make them behave as antimatter particles.

e) It may possess speed which is comparable to the speed of light, for which it may behave like a light beam

f) Since the electron-positron pair exists in reversible equilibrium with photons, the electrons and positrons may behave as massless particles similar to photons in electromagnetic waves,

g) It may behave as a constituent of a laser beam.

\section{Nuclear Quadrupole Moment.}

It is found that many nuclei possess quadrupole moments, equivalent to a system of four magnetic poles. The quadrupole moment would be zero if the positive charge on a nucleus is distributed in a completely symmetrical manner. The nonzero quadrupole moment implies that the positive charge on the nucleus is not symmetrically distributed. Equation (15) describes a string of infinite length, which is a polymer (-ud- $)_{\mathrm{x}}$ of the monomeric unit, (-ud-). For x odd, this polymer is unsymmetrical about its middle point; that is, if the polymer is folded in half, then one half will not be identical with, and indistinguishable from, the other. For example, for $\mathrm{x}=3$, the length of the string is given by ud-ud-ud=3ud which when folded in half gives $u d+u d / 2=u d+d$ or $u d+u$. Obviously, the two halves are not identical, suggesting that the nucleus is not symmetrical. The total charge on $u d+d$ is

equal to $+2 / 3-1 / 3-1 / 3=0$ and the total charge on $u d+u$ is equal to $-1 / 3+2 / 3+2 / 3=+1$. So, the distribution of positive charge on the nucleus of composition 3ud is not symmetrical. Further, the segment d-u-d possesses two negative poles at the ends and the segment $\mathrm{u}-\mathrm{d}-\mathrm{u}$ possesses two positive poles at the ends. In consequence, the nucleus $3 \mathrm{ud}$ is expected to possess quadrupole moment. The overall charge on the nucleus is equal to $3 x(+1 / 3)=+1$ which represents the deuterium nucleus. So, the deuterium nucleus (uud and udd) may possess quadrupole moment.

For $\mathrm{x}=6$, equation (15) produces a string of length equal to ud-ud-ud-ud-ud-ud $=6 \mathrm{ud}$. If the string is folded in half, then one obtains two halves of composition ud-ud-ud (or 3ud) each. It is obvious that the two halves are identical and indistinguishable; that is, the two halves appear as the same string, suggesting that the string ud-ud-ud-ud-ud-ud is symmetrical. The charge on the string ud-ud-ud is equal to $3 \times(1 / 3)=+1$ which is the same as that on the other half. This implies that the positive charge is symmetrically distributed on the nucleus. The total charge on the nucleus is equal to $+1+1=+2$ which represents the He- 4 nucleus ( 2 uud +2 udd). So, the He- 4 , nucleus is not supposed to possess quadrupole moment. It is, therefore, concluded that for $\mathrm{x}$ odd, equation (15) produces nuclei in which the positive charge is unsymmetrically distributed, for which they may possess quadrupole moment. But for $\mathrm{x}$ even the same equation produces nuclei in which the positive charge is symmetrically distributed, for which they may not possess quadrupole moment. 


\section{Conclusions.}

a. There are infinitely large number of nuclei of composition xud, where $u$ and $d$ are $u$ quark and d quark, respectively, and $\mathrm{x}=1,2,3, \ldots, \infty$

b. An energetic photon can clone itself in collision with a nuclear ud pair. This process may be used to construct a laser beam.

c. The members of the ud pair are acted upon by two kinds of force: One, gravitational force of attraction carried by a pair of photons (or an electron-positron pair) exchanged between $u$ and d. Two, the Coulombic force of attraction between the positively charged $u$ quark and the negatively charged $\mathrm{d}$ quark.

d. The energy of an exchanged photon is quantized.

e. The nuclei in which the positive charge is unsymmetrically distributed may possess quadrupole moment but those in which the positive charge is symmetrically distributed may not possess quadrupole moment.

f. If the universe expands and $\mathrm{h}$ remains constant, then the speed of light $\mathrm{c}$ decreases; but $\mathrm{h}$ increases if $\mathrm{c}$ remains constant. If both $\mathrm{h}$ and $\mathrm{c}$ are allowed to change, simultaneously, then $\mathrm{h}$ increases and $\mathrm{c}$ decreases. However, If both $\mathrm{h}$ and $\mathrm{c}$ are kept constant, then the universe remains static.

\section{References.}

Beiser, A. (1987). Concepts of Modern Physics, physics Series (4th ed., pp. 70-78). New York : McGraw-Hill.

Bhuiyan, A. L. (2013). Qualitative Nuclear Structures in Terms of Clusters of Quarks. Physics Essays, 26(2), 223-236. http:dx.doi.org/10.4006/0836-1398-26.2.223

Feynman, R. P., Leighton, R. B., \& Sands, M. (1988). The Feynman Lectures on Physics: Mainly Electromagnetism and Matter. Vol. II. P.42. Narosa, Indian Student Edition, New Delhi, India.

Glasstone, S. (1958). Sourcebook on Atomic Energy (3rd ed, pp. 87-90). London: Van Nostrand Co..

Goldman, T., Hughes, R. J., \& Nieto, M. M. (1988). Gravity and Antimatter. Scientific American, 258(3), 32-40.

Hawking, S. (1988). A Brief History of Time: From Big Bang to Black Holes (pp. 77-79). London: Bantam Books.

Sekine, M. (1985). The structure of the Nucleon. International Journal of Theoretical Physics, 24(7), 701-705. http.//dx.doi.org/10.1007/BF00670877

Sekine, M. (2012). The Cantor Set Constructed from an Infinite Number of Quarks Constituting the Nucleon and the Dark Matter. Applied Physics Research, 4(4), 68-72. http://dx.doi.org/10.5539/apr.v4n4p68

\section{Copyrights}

Copyright for this article is retained by the author(s), with first publication rights granted to the journal.

This is an open-access article distributed under the terms and conditions of the Creative Commons Attribution license (http://creativecommons.org/licenses/by/3.0/). 